\title{
WEIRD - Real Use Cases and Applications for the WiMAX Technology
}

\author{
Silvano Mignanti \\ University of Rome "Sapienza" \\ Rome, Italy \\ silvano.mignanti@dis.uniroma1.it \\ Mario Castellano, Vincenzo Augusti \\ Istituto Nazionale di Geofisica e Vulcanologia \\ Osservatorio Vesuviano - Naples, Italy \\ castellano@ov.ingv.it-augusti@ov.ingv.it \\ Giuseppe Martufi \\ Elsag Datamat \\ Rome, Italy \\ giuseppe.martufi@elsagdatamat.it
}

\author{
Pedro Miguel Neves \\ Portugal Telecom Inovação \\ Aveiro, Portugal \\ est-p-neves@ptinovacao.pt \\ Cinzia Mambretti \\ Socrate Medical \\ Milan, Italy \\ cinzia@socratemedical.it \\ Fausto Andreotti \\ Italtel \\ Milan, Italy \\ fausto.andreotti@italtel.it
}

\begin{abstract}
IEEE 802.16/WiMAX is one of the most promising technologies for Broadband Wireless Access, both for fixed and mobile use. This paper presents the structure of some testbeds, set up in the framework of the European project WEIRD, about novel applications running on top of a WiMAX-based end-to-end architecture. The presented testbeds are based on real use case scenarios, including monitoring of impervious areas, telemedicine and tele-hospitalization.
\end{abstract}

Keywords: WiMAX; environmental monitoring; testbed

\section{INTRODUCTION}

The WEIRD project [1], which started in June 2006 and has a duration of 2 years, will prototype 4 pan European testbeds able to support novel application scenarios, such as environmental monitoring, tele-medicine and fire prevention, making an efficient use of the fixed/portable (IEEE 802.16d) [2] and mobile (IEEE 802.16e) [3] WiMAX technology. The consortium is made up of a well balanced number of partners, from manufacturers, operators, system integrators, academia and users. In fact, it is the presence of final users as volcano monitoring observatories (Vesuvio and Iceland), telehospitalization centers (Istituto Oasi Maria S.S.) and forest fire prevention centers (ADAI) that is driving effectively the system requirements and will help the validation and exploitation phase of the project. WiMAX [4], as a broadband wireless access (BWA) technology, is able to provide ubiquitous internet access allowing end users to be connected to the internet independently of their location, even in rural and impervious areas where the deployment of a wired access solution would not be economically feasible. Here follows a set of example scenarios in which the WiMAX technology could play a significant role:

\section{A) Environmental Monitoring}

An effective and reliable monitoring system must be implemented to preview the occurrence of natural catastrophes. To achieve this aim, permanent and mobile stations (video-cameras and sensors) are installed for sending data in real time to an aggregation point using both Fixed and Mobile WiMAX; then, data is forwarded to the scientific community in the Monitoring Centre through a WiMAX backhaul. Furthermore, Mobile WiMAX is also used to allow real time communication between mobile users visiting the impervious area and the Monitoring Centre.

\section{B) Fire Prevention}

Several pilot projects have shown how the usage of technologies such as sensors, as well video and infrared cameras can help fire detection. Important issues associated to the implementation of such systems are the costs and the image quality provided by GSM/GPRS communications, as well as the difficulty to implement radio links to transmit video in mountainous regions. In WEIRD project the applications for fire prevention that take advantages of the WiMAX technology are related to:

- transmission of JPEG images and text data taken from the Forest Fire Simulation System located and operated in the District Civil Protection Coordination Centre (CC);

- communication between mobile units in the field and the $\mathrm{CC}$, as well as between the fixed/mobile video surveillance systems and the $\mathrm{CC}$;

- Transmission of data acquired from a network of sensors distributed in forest locations with hard accessibility, where ambient conditions can significantly vary.

Mobility support is crucial in these environments, requiring the implementation of fast handoff mechanisms and the provisioning of specific QoS levels in mobile environments

\section{C) Telemedicine}

E-health is one of the areas where WiMAX technologies can substantially contribute to improve the daily activities and thus enhance the quality of life. Remote diagnosis is one of the 
possible cases where WiMAX plays an important role. For instance, a doctor in an ambulance equipped with a portable ultrasound device, connected to a notebook, as well as to the hospital through a Mobile WiMAX channel, can intervene in a car accident. Besides the Remote Diagnosis scenario, Mobile WiMAX is also important for other Telemedicine applications such as Tele-hospitalization, Remote Follow-up, Remote Monitoring and Remote Assistance. The Telemedicine scenario requires real time services and applications such as voice and video over IP to support real time communication in emergency cases.

\section{APPLICATIONS}

In WEIRD, the following applications have been foreseen: A) Environmental Monitoring Applications - including fire and volcano monitoring, which make use of data and video streaming from sensor networks and cameras for the respective user communities.

B) Telemedicine Applications - including high-resolution video and data streaming from medical equipment, for the clinical user communities.

C) Generic User Applications - like VoIP and videoconference over IP, including also video-streaming for generic use in monitoring and content diffusion. In general, voice and video over IP applications will be used to test WEIRD solutions and to demonstrate the benefits offered by the WiMAX technology.

All these applications are QoS demanding and thus require the availability of a broadband channel and can hardly be used in mobility, especially in remote and impervious areas. These applications can be split into two groups: one using SIP for session setup and one not. Another grouping criterion is the possibility to adapt the code of the application to the WEIRD system - we will so distinguish between customizable and legacy applications. The WEIRD framework has been designed in order to support both.

Regarding SIP applications, a WEIRD SIP Proxy, located on the core service network side, is used for extraction of Service Description Information (SDI) from the SIP/SDP messages, resource reservation triggering toward the WEIRD control plane and interaction with AAA services as well. This approach is compliant to the one used in the IMS/TISPAN framework.

The WEIRD framework can support different QoS models (enabled or assured), also making it possible to deploy commercial-off-the-shelf (COTS) soft-phones. In addition, other WEIRD software modules running on the client side have been developed to support non-SIP-based applications: WEIRD agent(s) and WEIRD Application Programming Interfaces (APIs) will provide services like enhanced QoS and seamless mobility features for legacy and customizable applications, respectively.

\section{SCENARIOS}

WEIRD project is scenario-driven and therefore scenarios play a crucial and very important role in the system specification. Two challenging scenarios for the WiMAX technology have been foreseen to test the enhancements developed for the technology:

\section{A. Volcano Monitoring}

This scenario is critical; in fact the seismic monitoring is, probably, the most important method for the active volcanoes surveillance systems. The Istituto Nazionale di Geofisica e Vulcanologia-Osservatorio Vesuviano (INGV-OV) is a research institute having the task to monitor two high-risk densely populated active volcanic areas: Vesuvio and Campi Flegrei (near Napoli, southern Italy). The Campi Flegrei volcanic area has been characterized in the last years by slow ground deformation accompanied by low-energy swarm-type seismic activity [5]. The seismic monitoring is realized by means of a Permanent Seismic Network. Data is transmitted in real-time to an Acquisition Center using radio links or dedicated phone lines [6].

Notwithstanding this, to improve the monitoring system during a crisis, a Temporary Seismic Network is deployed to integrate the Permanent one (Fig.1). The Temporary Stations, placed where needed to improve the Permanent Network acquisition capabilities, gather the seismic signal in local mode, storing the data on high capacity hard disks. Data is periodically collected, analyzed and integrated with the Permanent Network ones. The integration of Permanent and Temporary Seismic Networks gives complete information about the seismic pattern occurring during a crisis. This information is essential to the possible eruption forecasting.

Moreover, in case of seismic or volcanic crises, it is very important to obtain as soon as possible the data of the Temporary Network, for example using a communication system that allows the download from the remote stations of all the acquired signals.

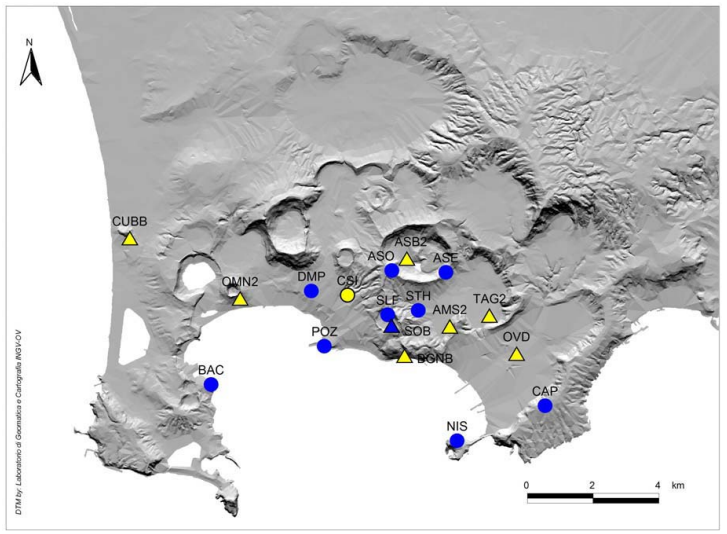

Figure 1. Permanent (blue symbols) and Temporary (yellow symbols) Seismic Networks operating in the "Campi Flegrei" volcanic area.

At present, the WiMAX technology is a very useful solution to download the acquired data from the remote Temporary Seismic Stations to the Acquisition Centre. In this way, the time lag between the acquisition of the data and their availability to the scientific community is sensibly reduced. Moreover, during seismic crises it allows to increase the data availability for analysis with near real-time procedures.

\section{B. Telemedicine}


E-health is one of the areas where WiMAX technologies can substantially contribute to improve the daily activities thus contributing to enhance the quality of life in Europe.

Today a large number of activities are carried out with limited success, unnecessary costs and human difficulties because of the impossibility to exchange real time information between different elements of the chain that are not at fixed locations. Here following some examples are listed of advanced medical services that wireless data and images transmission would make it possible:

- Remote diagnosis: need to transmit urgent data in order to make an immediate basic diagnosis, e.g. in occasion of street accidents, people in special health conditions (peacemaker bearers, pregnant women, etc). Two sub cases are envisaged:

$\circ$ Data are collected in a fix place,

$\circ$ Data are collected on a moving medium, e.g. an ambulance.

- Need to intervene on non transportable patients (e.g. accidents,) may require off-air transmission of critical data or images (e.g. last echography, PET, real-time video).

- Remote monitoring: today elder people are remotely monitored when at home, not when traveling.

- Remote follow-up: today patients travel to far-away hospitals to be followed-up after therapies or chirurgical interventions.

The telemedicine application is composed by different systems working together with the aim of helping remote doctor on the field in need of a second opinion. The remote doctor, sending images, video streaming and data collected from a portable medical device (e.g. Ultrasound system, Electrocardiography) to the hospital server should get the necessary support.

WiMAX technology is essential in the remote area, where the availability of guaranteed and cheap broadband channels may allow speeding up activities in case of emergency, thus saving human lives, as well as to save patients' time and travel costs to hospital in case of remote monitoring and follow-up activities.

\section{WEIRD ARCHITECTURE}

The WEIRD project is focused on the Radio Access Network (RAN), using WiMAX as the access technology. The defined architecture must be Next Generation (NGN) oriented and compliant with the main standardization bodies, such as the WiMAX Forum, the IETF 16ng Working Group (WG) [7] and the IEEE 802.16 standard. This implies that requirements, such as QoS, seamless mobility, authentication mechanisms, $\mathrm{IPv} 4 / \mathrm{v} 6$ support, as well as WiMAX equipment independency are guaranteed.

In order to be compliant with the WiMAX Forum, the specified architecture shall be in line with the Network Reference Model (NRM) defined by the Network Working Group (NWG). Thus, three main functional entities, as represented in Fig. 2, are specified: Customer Premise Equipment (CPE): The CPE might be fixed (IEEE 802.16d) or mobile (IEEE 802.16e). Access Service Network (ASN): The
ASN is the network entry point for the MS. It provides all the required functionalities, such as L2 connectivity, relay of AAA information to the MS home network, DHCP relay for allocation of an IP address to the MS, as well as micro and macro mobility support. The ASN is composed by two main logical entities: IEEE 802.16 Base Station (BS) and the ASNGW. The ASN-GW links the ASN with the CSN and acts as a gateway for the WiMAX network, as well as a control entity for the ASN; Connectivity Service Network (CSN): The CSN is responsible for providing IP connectivity to the MS (DHCP server), as well as for establishing the connectivity with other networks (IP backbone). Macro mobility scenarios are also supported by the CSN - MIP Home Agent (HA).

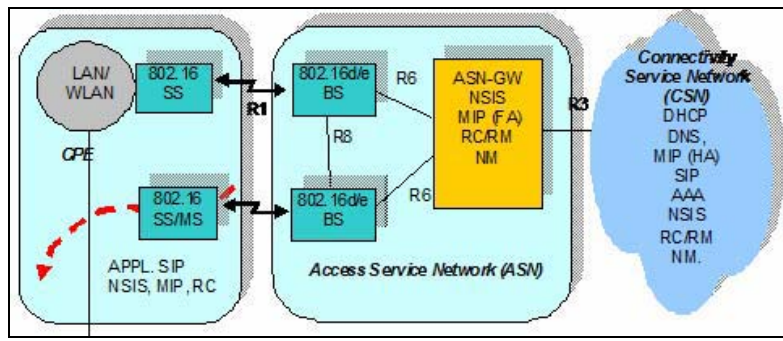

Figure 2. WEIRD Infrastructure

Fig. 3 provides a high level overview about the WEIRD architecture.



Figure 3. High Level WEIRD architecture

The presented architecture is able to cope with SIP and legacy applications. In the former case, a SIP UA (User Agent) is located on the MS and a SIP Proxy, acting as an 
Application Function (AF), is located in the CSN. The SIP Proxy intercepts the SIP signaling and provides the requested QoS parameters, extracted from the SDP messages, to the Connectivity Service Control (CSC) module on the ASN (CSC_ASN). An IMS compliant interface $(\mathrm{Gq} / \mathrm{Gq}$ ') is used to establish this communication. Additionally, the SIP Proxy exchanges information with the AAA server in order to perform authentication and authorization for the MS.

The CSC_ASN module is the main coordination point for QoS management and admission control mechanisms. The next step for the CSC_ASN is to perform Admission Control in both the ASN level and the WiMAX link. If the Admission Control process is successful, the CSC_ASN shall start QoS reservations in both the ASN and the WiMAX levels. In order to establish the QoS reservations in the WiMAX link, the CSC-ASN sends a QoS reservation request message to the Resource Controller (RC) module on the ASN-GW.

The $\mathrm{RC}$ is the first module of the architecture chain that is WiMAX technology dependent. Therefore, after receiving the QoS reservation request from the CSC_ASN, the RC will map the received generic QoS parameters to WiMAX specific QoS parameters and forward the reservation request to the WiMAX vendor dependent module - Adapter. Despite the RC is a WiMAX technology dependent module, it is independent of the specific WiMAX vendor equipment. This is the role of the Adapter and it is the only module that is necessary to implement to support a new WiMAX BS from a different vendor. To enforce the establishment of the QoS Service Flows and the related Classifier Rules on the WiMAX link, the Adapter uses the SNMP protocol.

For legacy applications, a specific module has been specified for the MS - WEIRD Agent (WA). This module is responsible for the extraction of the QoS parameters from the legacy application. The gathered information is sent to the CSC on the MS (CSC_MS) and the NSIS protocol is used to carry the QoS reservation request towards the CSC_ASN. Finally, the reservation process in both the ASN and the WiMAX link is similar to the one previously explained for the SIP applications case.

\section{WEIRD TESTBEDS}

One of the key tasks of WEIRD project is to validate and demonstrate the results. For this purpose, four testbeds have been implemented in Finland, Italy, Portugal and Romania, each one built around a specific scenario and employing specific technologies suitable for that scenario and its associated applications. The proposed framework has the capability to support different end-to-end signaling scenarios, like intra-domain and inter-domain communication.

Both fixed and mobile WiMAX solutions are evaluated by all the testbeds, mostly in the first and seconf phase, respectively. Each testbed is provided by one or more WiMAX access networks. As illustrated in Fig. 4, all testbeds are connected between each other through the different National Research Networks (NRENs) and the European Network GEANT2. Table 1 summarizes the main mobile WiMAX related demonstrations of the four testbeds
TABLE 1

KEY MOBILE DEMONSTRATIONS AND RELATED SCENARIOS

\begin{tabular}{|c|c|c|c|}
\hline $\begin{array}{l}\text { Testbed } \\
\text { Italy } \\
(3.5 \mathrm{GHz})\end{array}$ & $\begin{array}{c}\text { Demonstration: } \\
\text { - Mobility capabilities } \\
\text { - Full management } \\
\text { functionality } \\
\text { - LOS/NLOS } \\
\text { - RoF } \\
\end{array}$ & \multirow[t]{2}{*}{$\begin{array}{c}\text { Testbed } \\
\text { Portugal } \\
(3.5 \mathrm{GHz})\end{array}$} & $\begin{array}{c}\text { Demonstration: } \\
\text { - Mobile video surveillance } \\
\text { - A/V streaming } \\
\text { - LOS } \\
\text { - Real time data collection } \\
\text { and transmission } \\
\end{array}$ \\
\hline & Scenario: Telemedicine & & Scenario: Fire prevention \\
\hline $\begin{array}{l}\text { Testbed } \\
\text { Romania } \\
(3.5 \mathrm{GHz})\end{array}$ & $\begin{array}{c}\text { Demonstration: } \\
\text { - Mobility capabilities } \\
\text { - Full management } \\
\text { functionality } \\
\text { - LOS/NLOS } \\
\text { - VoIP }\end{array}$ & \multirow[t]{2}{*}{$\begin{array}{l}\text { Testbed } \\
\text { Finland } \\
(3.5 \mathrm{GHz})\end{array}$} & $\begin{array}{c}\text { Demonstration: } \\
\text { - Mobile capabilities } \\
\text { - Multimedia transmission } \\
\text { with error resilient coding } \\
\text { - Range extension } \\
\text { - Reliability }\end{array}$ \\
\hline & $\begin{array}{l}\text { Scenario: video conf., } \\
\text { surveillance, e-learning }\end{array}$ & & $\begin{array}{l}\text { Scenario: Environmental } \\
\text { monitoring }\end{array}$ \\
\hline
\end{tabular}

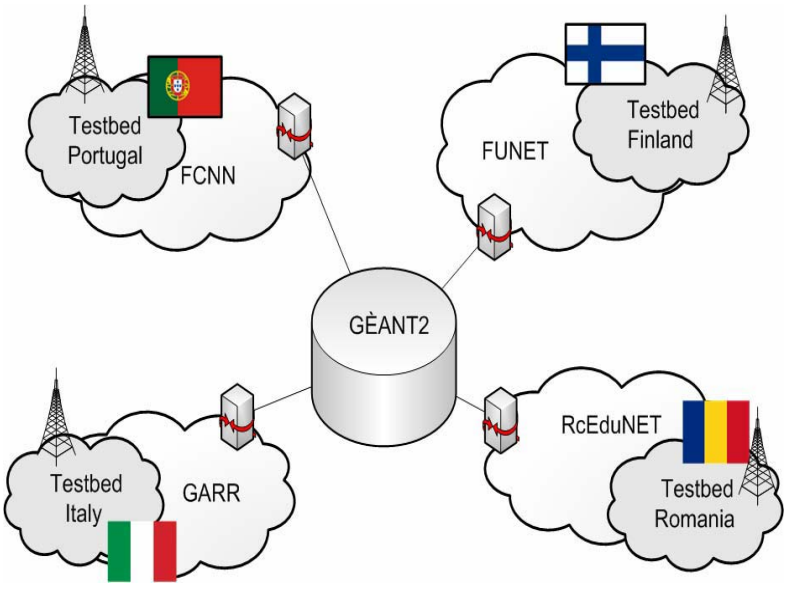

Figure 4. WEIRD testbeds

\section{WEIRD RESULTS}

A preliminary system prototype has been developed during the phase 1 of WEIRD project. The result is a distributed system which is able to support applications in the following target scenarios:

\section{A. Volcano Monitoring}

This demo is mainly related to WiMAX antenna remoting by using the Radio over Fiber (RoF) technology implemented in WEIRD. The proposed applications, volcano monitoring using seismic sensors and VoIP, allow information sharing among scientists located on different competence centers. RoF systems employ fiber optics to transport RF modulated signals for wireless applications. These have potential advantages in allowing transmission of WiMAX signals in their raw form to antennas at which no RF signal processing beyond amplification is required, which leads to a simplification of the transmission equipment. RoF technology entails the use of optical fiber links to distribute RF signals from a central location to Remote Antenna Units (RAUs). RoF makes it possible to centralize the RF signal processing functions in one shared location (Central Base Station), and then to use optical fiber, which offers low signal loss to distribute the RF signals to the RAUs. The WEIRD RoF module deals mainly 
with IEEE 802.16d signals in WEIRD testbed at Ivrea, where a $1.1 \mathrm{Km}$ monomode optical-fiber link is used.

From the volcano-monitoring point of view, the test performed using WiMAX technology has given relevant enhancement in data transmission. During the test, we used a digital seismic station Lennartz M24 (24 bit, 6 channels) equipped with a short period ( $1 \mathrm{~s})$ seismometer Lennartz LE3Dlite. The data acquisition is on-site on a high capacity hard disk. With the WiMAX connection, a signal time window of 5 minutes in compressed ASCII format has been downloaded in about 6 seconds. As comparison, a GSM connection downloads the same signal in about 15 minutes. This test shows how WiMAX technology provides very fast communication system, allowing a real-time processing of the remote data.

\section{B. Telemedicine}

The focus here is to extend research networks by WiMAX technology to reach impervious locations. In particular, the aim is to demonstrate the effectiveness of the WEIRD testbed network by an operational remote medical site, using applications like mobile ultrasound data scanning and acquisition and videoconference. Telemedicine application requires a high number of data and images transfer. The dimension of the images and data (specific medical files) should vary typically from $300 \mathrm{~Kb}$ to $10 \mathrm{Mb}$. Besides data transfer, VoIP and Video Conference applications are helpful to provide real time communication between the patient/doctor on the field and the remote experts.

Other peculiarities of the tele-medicine applications are data privacy, access control and service assurance. Data privacy is assured by adopting TLS (Transport Layer Security), using dedicated PKI (Public Key Infrastructure) and CA (Certification Authorities). Access control has to be adopted at application and network layer by leveraging on identity management system, as well as authentication and authorization infrastructures. Furthermore network provider has also to provide a controlled allocation system able to guarantee authorized access to network services resources.

Summarizing, Telemedicine requires a lot of bandwidth available, either in a nomadic or mobile scenarios, with quality of service assurance. Therefore, WiMAX technology is very useful for this type of environments.

\section{CONCLUSIONS}

The experiments carried out in WEIRD testbeds demonstrated how the WiMAX technology could be profitably used in several everyday applications.

In volcano monitoring applications, WiMAX technology proved to be very fast in downloading the acquired data from remote Temporary Seismic Stations: the time delay reduction between the data acquisition and their accessibility allows a near real-time processing in case of emergency. Moreover, WiMAX is able to cover distances of several tens of kilometers allowing connecting stations installed on a wide monitored area. This possibility increases the information on the seismic activity occurring during a crisis. Fast and reliable data availability could be very helpful to the scientific community allowing them to define realistic estimates for the volcanic eruption forecasting.

In telemedicine applications, WiMAX technology has many peculiarities that overcome the limitations of current technologies, especially in relation to critical parameters like bandwidth, access control and reliability of service either in fixed, nomadic, and mobility conditions.

The use of RoF technology, in cooperation with WiMAX, allows the centralization of the processing of the RF signal, so allowing equipment sharing, dynamic allocation of resources, and simplified system operation and maintenance. These benefits can translate into significant savings in system installation and operational costs, especially in wide-coverage broadband wireless communication systems, where a high density of BS is necessary. The advantages of RoF technology in coverage extension will be very useful for those application scenarios envisaged in WEIRD, where critical customers need a reliable connection that can't be disrupted due to, for instance, extreme weather conditions, temporary obstacles or natural disasters.

\section{ACKNOWLEDGMENT}

The authors would like to thank members of WEIRD team who supported this work by participating to WEIRD testbed setup.

\section{WEIRD DISCLAIMER}

The work described in this paper is based on the results of IST FP6 Integrated Project WEIRD. The project receives research funding from the European Community's Sixth Framework Programme. Apart from this, the European Commission has no responsibility for the content of this paper.

\section{REFERENCES}

[1]WEIRD project website, http://www.ist-weird.eu

[2] IEEE Std. 802.16-2004, "IEEE Standard for Local and Metropolitan Area Networks, Part 16: Air Interface for Fixed Broadband Wireless Access Systems", IEEE Standard 802.162004, October 2004.

[3]IEEE Std 802.16e-2005, "IEEE Standard for Local and Metropolitan Area Networks, Part 16: Air Interface for Fixed Broadband Wireless Access Systems - Physical and Medium Access Control Layers for Combined Fixed and Mobile Operation in Licensed Bands", IEEE Standard 802.16e-2005, February 2006.

[4] WiMAX Forum website, http://www.wimaxforum.org

[5] G. Saccorotti, F. Bianco, M. Castellano, and E. Del Pezzo, "The July-August 2000 seismic swarms at Campi Flegrei volcanic complex, Italy", Geophys. Res. Lett., 28, n.13, pp. 2525-2528, 2001

[6]M. Castellano, C. Buonocunto, M. Capello, and M. La Rocca, "Seismic surveillance of active volcanoes: the Osservatorio Vesuviano Seismic Network (OVSN - Southern Italy)", Seism. Res. Lett., 73, 2, pp. 177-184, 2002.

[7] IETF 16ng WG: IP over 802.16 Networks (16ng), http://www.ietf.org/html.charters/16ng-charter.html 